\title{
Propiedades psicométricas del inventario alemán de ansiedad ante los exámenes en universitarios chilenos
}

\author{
Felipe E. Guerra ${ }^{1^{*}}$ y Ricardo A. Jorquera ${ }^{2^{*}}$ \\ (1) Complejo Tecnológico de Aprendizaje, Universidad de Atacama, Copayapu 485, Copiapó-Chile \\ (correo-e: felipe.guerra@uda.cl). \\ (2) Facultad de Humanidades y Educación, Departamento de Psicología, Universidad de Atacama, Copayapu 485, \\ Copiapó-Chile (correo-e: ricardo.jorquera@uda.cl).
}

* Autor a quien debe ser dirigida la correspondencia.

Recibido Sep. 1, 2020; Aceptado Oct. 27, 2020; Versión final Nov. 11, 2020, Publicado Abr. 2021

\section{Resumen}

El presente estudio buscó describir las características psicométricas del Inventario Alemán de Ansiedad ante los Exámenes (GTAl-A, por sus siglas en inglés) adaptados al español en una muestra de estudiantes universitarios chilenos. El estudio, de tipo instrumental con diseño no experimental transversal y con alcance descriptivo, se efectuó en una muestra de 239 universitarios. Las técnicas de análisis de datos usadas fueron el estadígrafo Omega de McDonald, para evaluar confiabilidad, y el análisis factorial confirmatorio, para establecer la validez de constructo de la escala. La confiabilidad del instrumento resultó adecuada. Se observaron valores Omega de McDonald entre 0,81 y 0,91 en las cuatro dimensiones del instrumento. El análisis factorial confirmatorio es favorable, evidenciándose una aceptable bondad de ajuste. Se concluye que el Inventario Alemán de Ansiedad ante los Exámenes demuestra que sus propiedades psicométricas avalarían su uso para evaluar la ansiedad frente a las evaluaciones académicas en estudiantes universitarios chilenos que están comenzando sus carreras académicas.

Palabras clave: ansiedad; exámenes universitarios; propiedades psicométricas; test alemán de ansiedad

\section{Psychometric properties of the German inventory of test anxiety in Chilean university students}

\begin{abstract}
The present study sought to describe the psychometric characteristics of the German Test Anxiety Inventory (GTAI-A) adapted to Spanish on a sample of Chilean university students. The study was of instrumental type, with a non-experimental cross-sectional design, and a descriptive scope. The sample consisted of 239 university students. The McDonald's Omega statistic was applied to evaluate reliability and the confirmatory factor analysis was used to establish the scale's construct validity. The results show that reliability of the instrument was adequate. The Omega and McDonald values ranged between 0.81 and 0.91 in the four dimensions of the instrument. The confirmatory factor analysis is favorable, showing an acceptable goodness of fit. It is concluded that the GTAI-A meets the psychometric properties required to recommend its use to assess the anxiety that Chilean university students face during academic evaluations at the beginning of their academic careers.
\end{abstract}

Keywords: anxiety; university exams; psychometric characteristics; German anxiety test 


\section{INTRODUCCIÓN}

La ansiedad ante las evaluaciones (en adelante $\mathrm{AE}$ ) se hace recurrente dentro del ámbito educativo superior (Álvarez, Aguilar y Lorenzo, 2012), ya que los estudiantes constantemente se ven sometidos a la evaluación de sus aprendizajes, siendo este momento uno de los que más preocupación y ansiedad ocasiona en los estudiantes de nivel superior, pudiendo afectar su bienestar emocional a lo largo del desarrollo dentro de las diversas instituciones educativas y a todo nivel del ciclo educativo (Dominguez-Lara, 2018). Es en este contexto, donde su investigación y profundización se hace primordial para el diseño de intervenciones psicoeducativas que ayuden a estudiantes, profesores y directivos a abordar de una manera adecuada esta problemática. Esto se hace aún más relevante si se considera la relación mutua que existe entre ansiedad y rendimiento académico, variables que funcionan como un sistema interdependiente (Hodapp, 1989).

Se define la AE como la disposición a responder con alta ansiedad-preocupación en situaciones asociadas a las evaluaciones académicas (Gutiérrez y Avero, 1995; Spielberger, 1980). Este tipo de ansiedad puede generar dificultades en una satisfactoria ejecución de la evaluación académica (Ávila-Toscano, Hoyos, González y Cabrales, 2011), pudiendo llevar a no lograr los objetivos deseados (Furlan, Ferrero y Gallart, 2014), así como también, afectar a procesos cognitivos como la atención selectiva, concentración y memoria de trabajo, los cuales juegan un rol fundamental en la vida universitaria (Fernández-Castillo y Caurcel, 2015).

El inicio de la medición de la AE se remonta a la década de los cincuenta cuando Saranson y Mandler (1952) se dedican a estudiar el rendimiento académico y la ansiedad, generando un cuestionario llamado Test Anxiety Questionnaire (TAQ). Estos autores entendían la ansiedad como un constructo unidimensional, con manifestaciones cognitivas, físicas y conductuales. Posterior a este enfoque unitario se da paso al estudio de la AE a partir del modelo Dual de Liebert y Morris (1967) que concibieron la ansiedad como un constructo compuesto por un elemento cognitivo, Ilamado preocupación, y otro de tipo fisiológico - afectivo, conocido como emocionalidad. A raíz de este modelo se crea el que es considerado a nivel planetario como el más utilizado a la hora de medir la ansiedad (Chapell, et al., 2005) el Test Anxiety Inventory (TAl, Spielberger, 1980).

Con el avance de los estudios respecto a la ansiedad y a las propiedades psicométricas de los instrumentos anteriormente creados, se presentan diferentes críticas y observaciones realizadas a los modelos unidimensional y bidimensional que principalmente apuntan a que sus dimensiones se superponían (Everson et al. 1991; Zeidner y Nevo, 1992; Zimmer et al., 1992). En consecuencia, se comienza a tratar a la AE como un constructo multidimensional, donde Hodapp (1991) crea el TAI-G como una adaptación a población alemana del TAI de Spielberger (1980), concibiendo a la AE como un modelo con un factor de segundo orden que agrupa cuatro factores de primer orden: emocionalidad (percepción de activación fisiológica), preocupación (diversos pensamientos sobre las consecuencias de fracasar), falta de confianza (creencia negativa referente a la capacidad de rendir de manera adecuada el examen) e interferencia (pensamientos que generan bloqueo cognitivo y distracción). Este modelo ha tenido evidencia que confirma su estructura factorial, y con ello, la adecuada validez de constructo del instrumento propuesto (Keith et al., 2003), lo cual ha sido constatado de manera transcultural y en diferentes idiomas (Ringeisen et al., 2010) y en versiones resumidas del instrumento (Mowbray et al., 2015).

La adaptación al español del test TAI-G, realizada en población argentina, se conoce como GTAI-A (Heredia et al., 2008), la cual fue obtenida luego de realizar un primer análisis factorial exploratorio que conservó la estructura de cuatro dimensiones, pero que arrojó índices de consistencia interna moderados en algunas escalas. Es por esta razón que se vuelve a aplicar el instrumento a una nueva muestra de estudiantes y se realiza un segundo análisis factorial exploratorio que obtuvo niveles de consistencia interna adecuados, conservando su estructura de cuatro dimensiones, pero eliminando 2 ítems (4 y 30), quedando finalmente integrada por 28 ítems de los 30 originales planteados en el TAI-G. Con la finalidad de seguir estudiando y validando las propiedades psicométricas del GTAI-A. Piemontesi et al. (2012) realizaron un análisis factorial exploratorio y otro confirmatorio mediante parcelación de ítems, obteniendo que el test se ajusta de manera aceptable a los datos y es congruente con el modelo multidimensional de Hodapp, creando el GTAI-AR para identificar la ansiedad ante las evaluaciones en estudiantes universitarios.

En relación a la aplicación del test en el contexto educativo universitario destacan los trabajos realizados por Piemontesi y Heredia (2011) quienes realizaron una investigación para explorar las relaciones entre las dimensiones de la ansiedad frente a los exámenes, las estrategias de afrontamiento utilizadas en esta situación importante, la autoeficacia para el aprendizaje autorregulado y el rendimiento académico, utilizando el GTAl-A como instrumento de medida de la AE. En esta línea, Furlan et al. (2012), al usar este instrumento, detectaron que estudiantes universitarios con mayor ansiedad ante las evaluaciones recurren más frecuentemente a estrategias de aprendizaje basadas en la repetición y utilizan menos estrategias reflexivas. Además, poseerían mayor tendencia a la autoculpabilización y la rumiación autofocalizada, lo cual contrasta 
con los menos ansiosos, quienes se orientarían hacia el problema buscando su resolución o su aceptación y reevaluación en términos más positivos. Respecto a las dimensiones específicas del GTAI-A, observaron que la preocupación se correlacionaría positivamente con estrategias de repetición y regulación del esfuerzo y con estilos menos adaptativos de afrontamiento, mientras que las dimensiones de falta de confianza e interferencia se relacionarían negativamente con estrategias de aprendizaje reflexivo y crítico y positivamente con estilos poco eficaces para afrontar el estrés. Similares evidencias encontraron Piemontesi et al, (2012). Asimismo, Arana y Furlan (2016) observaron que la ansiedad ante los exámenes en estudiantes universitarios estaba fuertemente vinculada a la dimensión negativa del perfeccionismo, esto es, con la discrepancia percibida por los estudiantes entre las metas deseadas y las obtenidas. También observaron que estos perfeccionistas desadaptativos tendían a sufrir más ansiedad ante los exámenes que los perfeccionistas adaptativos, caracterizados con sujetos que tendrían una predilección por imponer altos estándares.

En el ámbito de la intervención, Furlan (2013) usó el test en un contexto psicoeducativo con estudiantes universitarios argentinos, donde buscaba disminuir la ansiedad ante los exámenes, la procrastinación académica e incrementar la autoeficacia regulatoria, usando el test GTAI-AR en la evaluación y comparación de la ansiedad ante las evaluaciones pre y post intervención. Dicho programa fomentaba el automonitoreo de las estrategias de aprendizaje implementadas por el estudiante, así como el aprendizaje de técnicas cognitivoconductuales de control de la ansiedad y el ensayo anticipado de exámenes, lográndose mejorías moderadas. En la misma línea, Furlan et al. (2015) usan este test para medir la efectividad de un programa de intervención para disminuir la AE en estudiantes universitarios de psicología y medicina, considerando para estos efectos la ansiedad ante las evaluaciones como una medida global, además de considerar los puntajes de cada una de sus cuatro dimensiones.

Al respecto, constatan una disminución de los niveles de $A E$ y procrastinación y un incremento en la autoeficacia regulatoria. De la misma forma, Furlan et al. (2019) utilizaron el GTAI-AR para evaluar la AE en una intervención grupal con estudiantes universitarios, donde se utilizaron técnicas cognitivo-conductuales, mindfulness y terapia de aceptación y compromiso, con la finalidad de disminuir la ansiedad frente a los exámenes orales. En esta intervención se constataron disminuciones estadísticamente significativas en la evaluación global y en las dimensiones emocionalidad, preocupación y falta de confianza de la escala de ansiedad frente a los exámenes. Las adecuadas propiedades psicométricas de este instrumento en población universitaria y los buenos resultados obtenidos con él en aplicaciones específicas para reducir la ansiedad ante las evaluaciones y la no existencia de un instrumento validado para estos fines, motivan la evaluación de las características de medición del GTAI-A en estudiantes chilenos de nivel educacional universitario.

\section{METODOLOGÍA}

Este estudio es de tipo instrumental, pues su objetivo es la evaluación de las propiedades psicométricas de un instrumento de evaluación (Ato et al. 2013). Su diseño de investigación es de tipo no experimental transversal descriptivo.

\section{Participantes}

El muestreo fue de tipo no probabilístico de tipo intencionado. La muestra quedó conformada por 239 estudiantes de primer año (Mujeres: $67,8 \%, n=162$; hombres: $32.2 \%, n=77$ ) de cinco facultades de una universidad pública del norte de Chile, quienes accedieron voluntariamente a contestar el instrumento de evaluación. Las edades de los participantes estaban comprendidas entre los 17 y 41 años (M=18,86; DE: 3,00 ). Dentro de los criterios de inclusión se consideran hombres y mujeres que estén matriculados en primer año de una carrera universitaria y la participación voluntaria de cada uno de ellos; en cuanto a los criterios de exclusión ser estudiante de otros niveles de las carreras y no estar matriculado en la universidad donde se realiza el estudio.

\section{Instrumento}

El instrumento utilizado fue la Adaptación al español del Inventario Alemán de Ansiedad Frente a los Exámenes TAIG (Hoddap, 1991) en población argentina denominado GTAl-A (Heredia et al., 2008), donde participaron 212 estudiantes (mujeres $83.5 \%$; varones $16.5 \% ; M=22,7$ años). Este instrumento está conformado por 28 ítems divididos en cuatro subescalas: emocionalidad ( 8 ítems, $\alpha=0.88$ ), preocupación (9 ítems, $\alpha=0.87$ ), falta de confianza ( 6 ítems de codificación inversa, $\alpha=0.86$ ) e interferencia ( 5 ítems, $\alpha=0.74$ ). El cual debe ser respondido con una escala Likert de cuatro opciones que va desde 1= Nunca a 4= Siempre.

\section{Procedimientos}

En cuanto al procedimiento de recolección de datos, este se realizó al finalizar un taller que pretendía favorecer la inserción a la vida universitaria de los estudiantes de primer año de una universidad pública del norte de Chile, a quienes se les solicitó su participación voluntaria y anónima. A todos ellos se les explicaron 
los objetivos de la investigación, el uso y resguardo de los datos. Todos firmaron un consentimiento informado. La aplicación del test fue de forma masiva en modalidad online, en tiempos asignados para cada carrera.

En relación a los análisis de datos, se constataron los estadísticos descriptivos de los ítems y de las escalas, y se estimó la confiabilidad de ellas a través del estadígrafo omega de McDonald. La confirmación de la estructura del instrumento se verificó por medio de análisis factorial confirmatorio (AFC). En este caso, se efectuó la estimación de bondad de ajuste del modelo mediante el método de mínimos cuadrados ponderados diagonalizados (DWLS). Los índices que se consideraron en el AFC fueron: $\mathrm{chi}^{2}$, chi²/gl, el Índice de Bondad de Ajuste (GFI), el Índice de Tucker-Lewis (TLI), y el Índice de Bondad de Ajuste Comparativo (CFI). Para estos índices, valores superiores a ,90 se consideran indicadores de un ajuste aceptable del modelo. Además, se analizó la raíz del residuo cuadrático promedio de aproximación (RMSEA), en donde, valores inferiores a ,08 son considerados aceptables. Sumado a esto, se constató la invarianza factorial del instrumento en la muestra de mujeres y hombres. Se usaron los mismos indicadores de ajustes para describir y comparar la invarianza configural, métrica, escalar y estricta. Posterior a este procedimiento se comparó las medias de los puntajes de hombres y mujeres en los cuatro factores del instrumento y en el instrumento total mediante el estadígrafo t de Student para muestras independientes. Finalmente, mediante percentiles se establecieron baremos interpretativos del instrumento para hombres y mujeres. Los análisis descriptivos, las comparaciones de medias y el cálculo de percentiles se realizaron a través del software SPSS 22. La estimación de los indicadores omega de McDonald, el análisis factorial confirmatorio y el análisis de invarianza se efectuó a través del software JASP 0.14

\section{RESULTADOS}

En primer lugar, se realizó un análisis de los ítems, el cual se describe en la tabla 1. Las medias más altas se observan en las preguntas que miden la dimensión preocupación, en donde, salvo la pregunta 5 , todas tienen medias superiores a 3 . Con respecto a los valores de asimetría y curtosis, en todos los casos los valores muestran valores absolutos inferiores a 1. Las preguntas que componen la dimensión denominada falta de confianza presentan un omega de McDonald igual a ,910. A su vez, todos los ítems contribuyeron a la consistencia interna de la escala, pues la eliminación de cualquiera de ellos no suponía un incremento en el coeficiente Omega. A su vez, todas las correlaciones ítem/subescala muestran valores superiores a ,700.

Tabla 1: Estadísticos descriptivos y confiabilidad según dimensiones del Inventario de ansiedad frente a los exámenes.

\begin{tabular}{|c|c|c|c|c|c|c|}
\hline Sub-escala & Item & $M(D S)$ & $\begin{array}{l}\text { Correlación } \\
\text { Item/sub-escala }\end{array}$ & $\begin{array}{l}\text { McDonald's al } \\
\text { eliminar el ítem }\end{array}$ & Asimetría & Curtosis \\
\hline \multirow{6}{*}{$\begin{array}{l}\text { Falta de Confianza } \\
(\omega=, 910 ; \text { IC } 95 \%=, 889-, 925)\end{array}$} & 1 & $2,13(, 691)$ & ,709 & ,899 & 202 &,- 070 \\
\hline & 7 & $2,15(, 688)$ &, 770 & ,891 &, 186 &,- 074 \\
\hline & 12 & $1,99(, 804)$ & ,775 & ,889 & ,406 &,- 448 \\
\hline & 19 & $2,24(, 804)$ & ,728 & ,898 & ,309 &,- 350 \\
\hline & 25 & $2,09(, 725)$ & ,758 & ,893 & ,332 &,- 004 \\
\hline & 28 & $2,19(, 619)$ & ,764 & ,893 &,- 042 &,- 300 \\
\hline \multirow{9}{*}{$\begin{array}{l}\text { Preocupación } \\
(\omega=, 850 ; \text { IC } 95 \%=, 815-, 873)\end{array}$} & 2 & $3,43(, 575)$ &, 510 & ,840 &,- 397 &,- 756 \\
\hline & 5 & $2,84(, 769)$ &, 506 & ,839 &,- 216 &,- 357 \\
\hline & 8 & $3,15(, 835)$ &, 572 & 831 &,- 631 &,- 410 \\
\hline & 9 & $3,18(, 669)$ &, 572 & ,833 &,- 473 & ,259 \\
\hline & 13 & $3,44(, 645)$ &, 547 & ,838 &,- 925 & ,644 \\
\hline & 16 & $3,57(, 567)$ &, 578 & ,835 &,- 901 &,- 191 \\
\hline & 20 & $3,30(, 716)$ &, 519 & ,841 &,- 924 & ,962 \\
\hline & 22 & $3,13(, 693)$ & ,642 & ,824 &,- 401 &,- 065 \\
\hline & 26 & $3,19(, 816)$ & ,648 & ,820 &,- 640 &,- 447 \\
\hline \multirow{8}{*}{$\begin{array}{l}\text { Emocionalidad } \\
(\omega=, 900 ; \text { IC 95\%=,878-,917) }\end{array}$} & 3 & $2,74(, 830)$ & ,654 & ,890 &,- 061 &,- 675 \\
\hline & 6 & $2,54(, 823)$ & ,662 & 890 & 031 &,- 529 \\
\hline & 11 & $2,28(, 840)$ & ,651 & ,890 & ,335 &,- 144 \\
\hline & 15 & $2,34(, 840)$ & ,698 & 887 &, 226 &,- 485 \\
\hline & 17 & $2,94(, 810)$ & ,705 & ,886 &,- 364 &,- 414 \\
\hline & 21 & $2,05(, 885)$ & 635 & ,892 &, 569 &,- 347 \\
\hline & 24 & $2,26(, 899)$ & ,750 & ,881 & ,329 &,- 611 \\
\hline & 27 & $2,74(, 854)$ & ,719 & ,884 &,- 179 &,- 625 \\
\hline \multirow{5}{*}{$\begin{array}{l}\text { Interferencia } \\
(\omega=, 811 ; \text { IC } 95 \%=, 767-, 845)\end{array}$} & 4 & $2,58(, 711)$ & 629 & ,768 & ,396 &,- 421 \\
\hline & 10 & $2,54(, 743)$ & 600 & ,772 & 172 &,- 336 \\
\hline & 14 & $2,41(, 789)$ & ,636 & ,760 & ,364 &,- 276 \\
\hline & 18 & $2,20(, 768)$ & ,502 & ,803 & ,426 & ,023 \\
\hline & 23 & $2,31(, 730)$ & ,615 & ,770 & ,306 &,- 033 \\
\hline
\end{tabular}


En el caso de la dimensión denominada preocupación esta presenta un omega de McDonald igual a ,850. A su vez, todos los ítems contribuyeron a la consistencia interna de la escala y sus correlaciones ítem/subescala evidencian valores superiores a ,500. En la dimensión llamada emocionalidad se observa un omega de McDonald igual a ,900. En esta subescala todos los ítems contribuyeron a la consistencia interna de la escala y se aprecia que las correlaciones ítem/subescala presentan valores superiores a ,600. A su vez, las preguntas que componen la dimensión interferencia muestran un omega de McDonald igual a ,811. Igual que en las dimensiones anteriores, todos los ítems contribuyen a la consistencia interna de la escala, y las correlaciones ítem/subescala poseen valores superiores a ,500.

\section{Análisis factorial confirmatorio}

En primer lugar, se evaluó la normalidad multivariada a través de un análisis Mardia para la asimetría y curtosis multivariada, donde se halló un coeficiente de asimetría de 123,414, gl $=3654, \mathrm{p}<, 001$ y un coeficiente de curtosis de 824,579, $\mathrm{p}<$, 001, por lo cual queda demostrada la ausencia de una distribución normal multivariada de los datos. Por esta razón, además del carácter ordinal de las preguntas del instrumento, las cuales tenían un sistema de respuesta tipo Likert, se optó por realizar un análisis factorial confirmatorio usando un procedimiento basado en el método de mínimos cuadrados ponderados diagonalizados (DWLS). El modelo que se buscó confirmar fue el propuesto por Heredia et al. (2008), conformado por 28 preguntas distribuidas en cuatro factores.

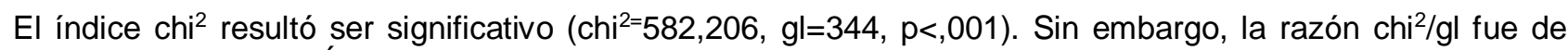
1,692, inferior a 3. El Índice de Bondad de Ajuste (GFI), que mide la cantidad relativa de varianza explicada por el modelo, mostró un valor adecuado (GFI=,995). También fueron buenos los valores obtenidos para el Indice de Tucker-Lewis $(\mathrm{TLI}=, 970)$ y el Índice de Bondad de Ajuste Comparativo (CFI=,973). El ajuste del modelo resultó también positivo al considerar la cantidad global de error existente en el modelo, pues el Root Mean Square Error of Aproximation presentó un valor inferior a 0,06 (RMSEA=,054; IC 90\%=,046-,061).

Tabla 2: Cargas Factoriales Estandarizadas

\begin{tabular}{|c|c|c|c|c|}
\hline & \multicolumn{4}{|c|}{ Componente } \\
\hline & F1 & F2 & F3 & $\mathrm{F} 4$ \\
\hline 1. Tengo seguridad en mi capacidad. &, 742 & & & \\
\hline 7. Tengo confianza en mi propio desempeño. &, 791 & & & \\
\hline 12. Sé que puedo confiar en mí mismo. & 819 & & & \\
\hline 19. Me siento conforme conmigo mismo. &, 810 & & & \\
\hline 25. Confío que lograré hacer todo. &, 759 & & & \\
\hline 28. Estoy convencido de que haré bien el examen. & ,833 & & & \\
\hline 2. Pienso en la importancia que tiene el examen para mí. & & ,386 & & \\
\hline 5. Me preocupa saber si podré afrontarlo. & & 854 & & \\
\hline 8. Pienso en las consecuencias de fracasar. & & 635 & & \\
\hline 9. Me pregunto si mi rendimiento será lo suficientemente bueno. & & 676 & & \\
\hline 13. Pienso en lo mucho que me importa obtener un buen resultado. & & ,328 & & \\
\hline 16. Me preocupo por el resultado de mi examen. & & ,437 & & \\
\hline 20. Me preocupa cómo se verá mi calificación. & & ,436 & & \\
\hline 22. Me preocupa que algo pueda salirme mal. & &, 774 & & \\
\hline 26. Pienso en lo que pasará si me va mal. & & 688 & & \\
\hline 3. Tengo una sensación rara en mi estómago. & & &, 695 & \\
\hline 6. Siento mi cuerpo tensionado. & & & ,712 & \\
\hline 11. Me siento incómodo. & & &, 768 & \\
\hline 15. Siento que mi corazón me late fuerte. & & & 679 & \\
\hline 17. Me siento ansioso. & & &, 746 & \\
\hline 21. Tiemblo de nerviosismo. & & &, 619 & \\
\hline 24. Tengo una sensación de angustia. & & & ,838 & \\
\hline 4. De repente se me cruzan pensamientos por la cabeza que me bloquean. & & &, 745 & \\
\hline 10. Pienso en cualquier cosa y me distraigo. & & & & ,932 \\
\hline 14. Fácilmente pierdo el hilo de mis pensamientos. & & & &, 427 \\
\hline $\begin{array}{l}\text { 18. Otros problemas personales me interfieren y no me permiten recordar las } \\
\text { cosas. }\end{array}$ & & & &, 685 \\
\hline $\begin{array}{l}\text { 23. Me llama la atención algo de poca importancia que interrumpe mi } \\
\text { razonamiento. }\end{array}$ & & & & 650 \\
\hline 27. Me siento nervioso. & & & &, 578 \\
\hline Covarianza entre los Factores & & & & \\
\hline $\mathrm{F} 1$ & & & & \\
\hline F2 &,- 363 & & & \\
\hline F3 &,- 578 & ,719 & & \\
\hline $\mathrm{F} 4$ &,- 488 & 416 &, 634 & \\
\hline
\end{tabular}


La tabla 2 muestra las cargas factoriales estandarizadas de los ítems y las covarianzas entre los cuatro factores que componen el instrumento. Las cargas factoriales de la gran mayoría de los ítems son apropiadas, llamando especialmente la atención las bajas cargas de los ítems 2 y 13, las cuales son inferiores a ,400. Por su parte, las covarianzas entre los factores son de moderadas a bajas, salvo entre los factores preocupación y emocionalidad, la cual llega a ,719.

\section{Invarianza Factorial según sexo}

Como se aprecia en la tabla 3, los índices de ajuste orientados a determinar la invarianza factorial del instrumento en la muestra de hombres y mujeres evidencian valores que permiten reconocer su equivalencia en ambos grupos. Los ajustes del modelo con el cual se determinó la invarianza configural mostró adecuados valores $(\mathrm{GFI}=, 993 ; \mathrm{CFI}=, 990$; $\mathrm{RMSEA=,032).} \mathrm{Igual} \mathrm{panorama} \mathrm{se} \mathrm{aprecia} \mathrm{en} \mathrm{los} \mathrm{valores} \mathrm{que} \mathrm{representan} \mathrm{el}$ ajuste de la invarianza métrica ( $\mathrm{GFI}=, 993 ; \mathrm{CFI}=, 983$; $\mathrm{RMSEA}=, 042)$, escalar (GFI=,992; $\mathrm{CFI}=, 984$; RMSEA $=, 039$ ) y estricta $(\mathrm{GFI}=, 992 ; \mathrm{CFI}=, 985 ; \mathrm{RMSEA}=, 037)$. Asimismo, destaca que las diferencias de los valores CFI de todos los modelos no difieren en más de, 01 .

Tabla 3: Índices de bondad de ajuste de los modelos puestos a prueba en la invarianza factorial

\begin{tabular}{|l|l|l|l|r|r|l|}
\hline & \multicolumn{1}{|c|}{$\mathrm{Chi}^{2}$} & \multicolumn{1}{c|}{ gl } & \multicolumn{1}{c|}{ GFI } & \multicolumn{1}{c|}{ CFI } & \multicolumn{1}{c|}{ TLI } & RMSEA \\
\hline Invarianza Configural & 766,325 & 684 &, 993 &, 990 &, 989 & $\begin{array}{l}, 032 \\
\text { IC } 90 \%=, 015-, 044\end{array}$ \\
\hline Invarianza Métrica & 856,215 & 708 &, 993 &, 983 &, 982 & $\begin{array}{l}, 042 \\
\text { IC } 90 \%=, 031-, 052\end{array}$ \\
\hline Invarianza Escalar & 871,275 & 736 &, 992 &, 984 &, 984 & $\begin{array}{l}, 039 \\
\text { IC } 90 \%=, 027-, 049\end{array}$ \\
\hline Invarianza Estricta & 890,359 & 764 &, 992 &, 985 &, 985 & $\begin{array}{l}, 037 \\
\text { IC } 90 \%=, 025-, 048\end{array}$ \\
\hline
\end{tabular}

Contraste de medias de los factores entre mujeres y hombres y Baremos

La tabla 4 muestra los estadísticos descriptivos de los puntajes de cada una de las dimensiones y para los puntajes totales del instrumento. Al respecto, se observan diferencias estadísticamente significativas entre hombres y mujeres en la dimensión emocionalidad $(t=4,419, p<, 01)$ y en el puntaje total $(t=3,023, p<, 01)$, en ambos casos las mujeres evidencian medias más altas. En la tabla 5 se presentan diferenciados por sexo los puntajes brutos de cada una de las dimensiones asociados a sus respectivos percentiles interpretativos.

Tabla 4: Descriptivos y comparación de medias de dimensiones y puntaje total del Inventario de ansiedad frente a los exámenes

\begin{tabular}{|l|l|l|l|l|l|l|l|}
\hline \multicolumn{2}{|c|}{ Sexo } & $\mathrm{N}$ & Media & $\begin{array}{l}\text { Desviación } \\
\text { estándar }\end{array}$ & $\mathrm{t}$ & $\mathrm{gl}$ & $\mathrm{p}$ \\
\hline \multirow{2}{*}{ Falta de Confianza } & Mujeres & 162 & 12,963 & 3,523 & 1,016 & 237 &, 311 \\
\cline { 2 - 9 } & Hombres & 77 & 12,456 & 3,806 & & & \\
\hline Preocupación & Mujeres & 162 & 29,580 & 4,067 & 1,951 & 237 &, 052 \\
\cline { 2 - 9 } & Hombres & 77 & 28,442 & 4,517 & & & \\
\hline \multirow{2}{*}{ Emocionalidad } & Mujeres & 162 & 20,877 & 4,717 & 4,419 & 237 &, 000 \\
\cline { 2 - 9 } & Hombres & 77 & 17,844 & 5,434 & & & \\
\hline Interferencia & Mujeres & 162 & 12,142 & 2,709 &, 830 & 237 &, 407 \\
\cline { 2 - 9 } & Hombres & 77 & 11,818 & 3,038 & & & \\
\hline Ansiedad Total & Mujeres & 162 & 75,562 & 11,328 & 3,023 & 237 &, 003 \\
\cline { 2 - 9 } & Hombres & 77 & 70,558 & 13,185 & & & \\
\hline
\end{tabular}

Tabla 5: Baremos del Inventario de ansiedad frente a los exámenes

\begin{tabular}{|c|l|l|l|l|l|l|l|l|l|l|}
\hline & \multicolumn{2}{c}{$\begin{array}{c}\text { Falta de } \\
\text { Confianza }\end{array}$} & \multicolumn{2}{c|}{ Preocupación } & \multicolumn{2}{c|}{ Emocionalidad } & \multicolumn{2}{c|}{ Interferencia } & \multicolumn{2}{c|}{ Ansiedad Total } \\
\hline Percentiles & Mujer & Hombre & Mujer & Hombre & Mujer & Hombre & Mujer & Hombre & Mujer & Hombre \\
\hline 10 & 8 & 8 & 24 & 21 & 15 & 12 & 9 & 8 & 61 & 55 \\
\hline 20 & 10 & 10 & 26 & 25 & 17 & 13 & 10 & 10 & 65 & 59 \\
\hline 30 & 11 & 11 & 28 & 26 & 18 & 14 & 10 & 10 & 69 & 62 \\
\hline 40 & 12 & 11 & 29 & 28 & 20 & 16 & 11 & 10 & 72 & 66 \\
\hline 50 & 13 & 12 & 30 & 29 & 21 & 17 & 12 & 11 & 76 & 70 \\
\hline 60 & 13 & 12 & 31 & 30 & 22 & 19 & 13 & 12 & 79 & 74 \\
\hline 70 & 14 & 13 & 32 & 31 & 23 & 21 & 14 & 13 & 82 & 78 \\
\hline 80 & 16 & 16 & 34 & 32 & 25 & 23 & 14 & 14 & 85 & 82 \\
\hline 90 & 18 & 19 & 35 & 34 & 28 & 25 & 16 & 16 & 90 & 89 \\
\hline
\end{tabular}




\section{DISCUSIÓN}

El propósito del presente estudio fue describir las características psicométricas de la adaptación Argentina del Inventario Alemán de Ansiedad frente a los Exámenes (Hoddap, 1991; Heredia et al., 2008) en estudiantes chilenos. Los resultados avalan las propiedades psicométricas del cuestionario. Se aprecia una adecuada confiabilidad de las subescalas que lo componen, y a su vez se confirma la estructura factorial evidenciada por los autores originales. En este sentido, se aprecia una adecuada bondad de ajuste del modelo de cuatro factores del instrumento. Junto a lo anterior, todos los factores del instrumento mostraron valores de confiabilidad Omega de McDonald adecuados, siendo todos ellos superiores a ,800.

Respecto a los baremos interpretativos, los resultados del presente estudio coinciden con las diferencias de género a nivel de la ansiedad total mostrada en la muestra argentina (Heredia et al., 2008). En ambos casos, el grupo de mujeres obtuvo medias más altas. Sin embargo, a nivel de dimensiones, en la muestra chilena se observan diferencias significativas entre hombres y mujeres solo en emocionalidad, mientras que en la muestra argentina, también se evidenciaron diferencias en preocupación y en falta de confianza. Importante sería profundizar en el estudio de posibles diferencias idiosincráticas en estos aspectos.

Es relevante contar con instrumentos validados que favorezcan la detección de ansiedad ante las evaluaciones de estudiantes universitarios chilenos, pues se ha observado que altos niveles de esta variable pueden generar dificultades de rendimiento académico y de bienestar emocional (Cassady, 2004). En este sentido, conocer con precisión los niveles de ansiedad de un estudiante, describiendo los niveles de cada una de las dimensiones que lo componen (falta de confianza, preocupación, emocionalidad e interferencia), permitirían realizar intervenciones pertinentes y acotadas a sus necesidades. Al respecto, se podrían obtener los réditos académicos deseados, a través del fomento de la utilización de estrategias de afrontamiento adaptativas, que no se orienten a la evitación, sino más bien se centren en el problema y/o en las emociones generadas por la evaluación académica (Dominguez-Lara, 2017).

Estas intervenciones también podrían focalizarse en el uso de estrategias de aprendizaje que se han visto relacionadas con menores niveles de ansiedad, como lo son el uso de la reflexión, en contraposición a estrategias basadas en la repetición. Esto cobra sentido, al observar que mayores niveles de preocupación estarían relacionados con el uso de estrategias de repetición, así como, la falta de confianza y la interferencia se asociarían negativamente con estrategias de aprendizaje reflexivo y crítico (Furlan et al., 2012; Piemontesi et al., 2012)

Otro aspecto positivo de tener un instrumento validado, que permita la evaluación de la ansiedad en el espacio académico, es que es posible utilizarlo para determinar la eficacia de intervenciones psicoeducativas orientadas a favorecer el manejo de la AE en estudiantes universitarios. Lo anterior permite que se puedan replicar intervenciones con demostrada efectividad (Furlan et al., 2019). Dentro de las limitaciones del presente estudio se encuentra el tamaño de la muestra y las características sociodemográficas de la misma. En general, en este tipo de estudios sería relevante contar con tamaños de muestras más numerosas, que representen diferentes niveles socioeconómicos, distintas regiones del país y diversos niveles académicos, con el fin de contrastar el comportamiento de este instrumento en poblaciones universitarias disímiles.

\section{CONCLUSIONES}

De acuerdo a los resultados obtenidos en este estudio se puede extraer la siguiente conclusión: el Inventario Alemán de Ansiedad frente a los Exámenes (28 items) demuestra que sus propiedades psicométricas avalarían su uso para evaluar la ansiedad frente a las evaluaciones académicas en estudiantes universitarios chilenos que están comenzando sus carreras académicas. Sin embargo, es recomendable seguir evaluando la dimensionalidad del instrumento, incluyendo muestras más amplias que consideren estudiantes de diferentes niveles y universidades.

\section{REFERENCIAS}

Arana, F. y Furlan, L., Groups of Perfectionists, Test Anxiety, and Pre-exam Coping in Argentine Students, doi: https://doi.org/10.1016/j.paid.2015.11.001, Personality and Individual Differences, 90, 169-173 (2016).

Álvarez, J., Aguilar J. y Lorenzo J., Test anxiety in university students: Relationships with personal and academic variables, Electronic Journal of Research in Educational Psychology, 10(1), 333-354 (2012).

Ato, M., López J. y Benavente A., Un Sistema de Clasificación de los Diseños de Investigación en Psicología, doi: https://dx.doi.org/10.6018/analesps.29.3.178511, Anales de Psicología, 29(3), 1038-1059 (2013).

Avila-Toscano, J., Hoyos S., González, D. y Cabrales A., Relación entre Ansiedad ante los Exámenes, Tipos de Pruebas y Rendimiento Académico en Estudiantes Universitarios, Psicogente, 14(26), 255-268 (2011).

Cassady, J. C., The Impact of Cognitive Test Anxiety across the Learning Testing Cycle, https://doi.org/10.1016/j.learninstruc.2004.09.002, Learning and Instruction, 14, 569-592 (2004). 
Chapell, M. S., Blanding Z.B., y otros cinco autores, Test Anxiety and Academic Performance in Undergraduate and Graduate Students, Journal of Educational Psychology, 97(2), 268-274 (2005).

Dominguez Lara, S., Procrastinación Académica, Afrontamiento de la Ansiedad Pre-Examen y Rendimiento Académico en Estudiantes de Psicología: Análisis Preliminar, doi: https://doi.org/10.24265/cultura.2017.v31.10, Cultura, 31, 181-193 (2017).

Dominguez Lara, S., Afrontamiento ante la Ansiedad Pre-Examen y Autoeficacia Académica en Estudiantes de Ciencias De La Salud, doi: https://doi.org/10.1016/j.edumed.2016.07.007, Educación Médica, 19, 39-42 (2018).

Everson, H., Millsap R.E. y Rodriguez C.M., Isolating gender differences in test anxiety: A Confirmatory Factor Analysis of the Test Anxiety Inventory, Educational and Psychological Measurement, 51, 243-251 (1991).

Fernández-Castillo, A. y Caurcel M. J., State Test-Anxiety, Selective Attention and Concentration in University Students, doi: http://dx.doi.org/10.1002/ijop.12092, International Journal of Psychology, 50(4), 265 - 271 (2015)

Furlan, L.,Sanchez, J., y otros tres autores. Estrategias de Aprendizaje y Afrontamiento en Estudiantes con Elevada Ansiedad frente a los Exámenes, Anuario de Investigaciones de la facultad de Psicología, 1(1), 130-141 (2012)

Furlan, L. A., Eficacia de una Intervención para Disminuir la Ansiedad frente a los Exámenes en Estudiantes Universitarios Argentinos, doi:http://dx.doi.org/10.15446/rcp, Revista Colombiana de Psicología, 22(1),75-89 (2013).

Furlan, L., Ferrero M. y Gallart G., Ansiedad ante los Exámenes, Procrastinación y Síntomas Mentales en Estudiantes Universitarios, doi:https://doi.org/10.32348/1852.4206.v6.n3., Revista Argentina de Ciencias del Comportamiento, 6(3), 31-39 (2014)

Furlan, L., Piemontesi S., Heredia D. y Sanchez-Rosas J., Ansiedad ante los Exámenes en Estudiantes Universitarios: Correlatos y Tratamiento, Anuario de Investigaciones de La Facultad de Psicología, 2(1), 37-53 (2015).

Furlan, L., Alonso-Crespo A., y otros tres autores, Tratamiento Grupal para la Ansiedad y la Evitación Conductual en Exámenes Orales, doi:https://doi.org/10.33898/rdp.v30i113.272, Revista De Psicoterapia, 30(113), $239-258$ (2019).

Gutiérrez, M. y Avero P., Ansiedad, Estrategias Auxiliares y Comprensión Lectora: Déficit de Procesamiento versus Falta de Confianza, Psicothema, 7, 569-578 (1995).

Heredia, D., Piemontesi S., Furlan L. y Volker H., Adaptación del Inventario Alemán de Ansiedad frente a los Exámenes: GTAI-A, doi: https://doi.org/10.35670/1667-4545.v8.n1.504, Revista Evaluar, 8(1) (2008).

Hodapp, V. Anxiety, Fear of Failure, and Achievement: Two Path-Analytical Models. doi: https://doi.org/10.1080/08917778908248727, Anxiety Research, 1(4), 301-312 (1989)

Hodapp, V., The Test Anxiety Inventory TAI-G: An Expanded and Modified Version with Four Components, Zeitschrift für Pädagogische Psychologie, 5, 121-130 (1991).

Keith, N., Hodapp, V., Schermelleh-Engel, K., y Moosbrugger, H. Cross-Sectional and Longitudinal Confirmatory Factor Models for the German Test Anxiety Inventory: A Construct Validation, doi: https://doi.org/10.1080/1061580031000095416, Anxiety, Stress, \& Coping, 16(3), 251-270 (2003)

Liebert, R.M. y Morris L.W., Cognitive and Emotional Components of Test Anxiety: A Distinction and Some Initial Data, Psychological Reports, 20, 975-978 (1967).

Mowbray, T., Jacobs, K. y Boyle, C., Validity of the TAI-G in an Australian Sample. doi:10.1111/ajpy.12058, Australian Journal of Psychology, 67, 121-129 (2015)

Piemontesi, S. y Heredia D., Relaciones entre la Ansiedad frente a los Exámenes, Estrategias de Afrontamiento, Autoeficacia para el Aprendizaje Autorregulado y Rendimiento Académico, Revista Tesis, 1, 74-88 (2011).

Piemontesi, S., Heredia D. y Furlan L., Propiedades Psicométricas de la Versión en Español Revisada del Inventario Alemán de Ansiedad ante Exámenes (GTAl-AR) en Universitarios Argentinos, doi:https://doi.org/10.11144/Javeriana.upsy11-1.ppve, Universitas Psychologica, 11(1), 177-186 (2012).

Piemontesi, S., Heredia, D., y otros tres autores, M. Ansiedad ante los Exámenes y Estilos de Afrontamiento ante el Estrés Académico en Estudiantes Universitarios. Anales De Psicología, 28(1), 89-96 (2012)

Ringeisen, T., Buchwald, P., \& Hodapp, V., Capturing The Multidimensionality of Test Anxiety in Cross-Cultural Research: An English Adaptation of the German Test Anxiety Inventory. Cognition, Brain, Behavior: An Interdisciplinary Journal, 14(4), 347-364 (2010)

Sarason, S.B. y Mandler G., Some Correlates of Test Anxiety. Journal of Consulting and Clinical Psychology, 47, 810-817 (1952).

Spielberger, C.D., Test Anxiety Inventory: Preliminary Professional Manual, Consulting Psychologists Press, Palo Alto, CA (1980).

Zeidner, M. y Nevo B., Test Anxiety in Examines in a College Admission Testing Situation: Residence, Dimensionality, And Cognitive Correlates, Advances in Test Anxiety Research, 7, 288-303 (1992).

Zimmer, J., Hocevar D., Bachelor P. y Meinke D.L., Art Analysis of the Sarason (1984) Four-Factor Conceptualization of Test Anxiety, Advances in Test Anxiety Research, 7, 103-113 (1992). 